\title{
Control of a Magnetic Fluid Drop Moving inside a Cylinder
}

\author{
M. Shinohara \\ Gifu National College of Technology, Gifu, 501- 0495 Japan \\ Email: sinohara@gifu-nct.ac.jp
}

\begin{abstract}
An investigation has been made of the dynamic behavior of a magnetic fluid drop settling in a viscous fluid inside a cylinder in response to the imposed magnetic fields. Two coils placed with their axies coincident with the cylinder axis provide the magnetic field for the tests. The Reynolds number $R e$ $\left(R e=a v_{z} / \nu\right)$ is in the range of $0.001 \sim 0.037$. Here, $a$ is the radius of the magnetic fluid drop, $v_{z}$ is the settling velocity of the drop and $\nu$ is the kinematic viscosity of machine oil.

Figure shows the shapes of a magnetic fluid drop of volume $1.12 \mathrm{~mm}^{3}$ settling in the machine oil inside a cylinder. The right figure shows the deformation of a magnetic fluid drop under the influence of a magnetic field. The current supply to two coils was $2 \mathrm{~A}$. Near the upper and lower coils, the drop is elongated. This deformation reduced the drag of the drop and then accelerated it. Near the upper coil the center of the drop is forced upwards. However, at that place the velocity of magnetic fluid drops with volume 0.82 and $2.00 \mathrm{~mm}^{3}$ were slower than those at middle point. At the middle point between two coils, the drop tends to extend along the field. Due to the change of shape, the drag on the drop is reduced and the velocity of the drop is increased. Near the lower coil, however, the center of the drop is forced downwards, for the extension of the drop and the drag reduction, the velocity of the drop is increased significantly.

The left figure shows the experiment under no magnetic field. If the magnetic fluid drop is small enough, the shape of it is almost spherical and the drop settled with the constant velocity.
\end{abstract}

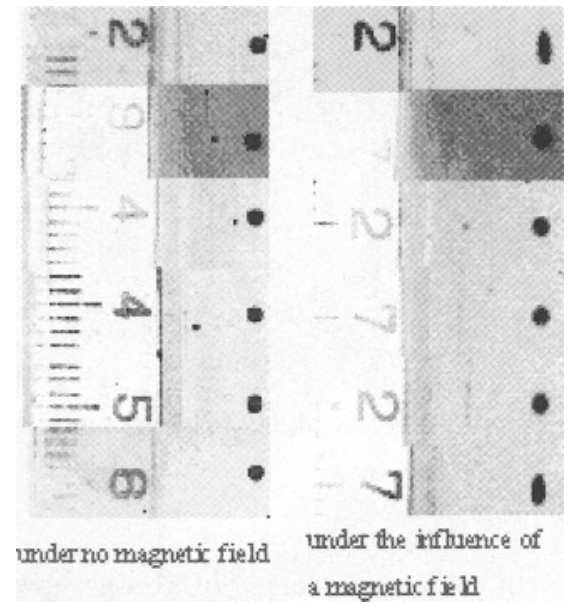

Figure: Shapes of a settling magnetic fluid drop (volume $1.12 \mathrm{~mm}^{3}$ ) 